\title{
ENDPOINTS OF THE SPECTRUM OF PERIODIC OPERATORS ARE GENERICALLY SIMPLE*
}

\author{
FRÉDÉRIC KLOPP ${ }^{\dagger}$ AND JAMES RALSTON ${ }^{\ddagger}$
}

\begin{abstract}
In this note, we prove that the edges of the spectral bands of a periodic Schrödinger operator are generically simple.
\end{abstract}

RÉSUMÉ. Cette note est consacrée à la preuve de ce que, pour un opérateur de Schrödinger périodique générique, les bord de bandes spectrales sont simples.

0. Introduction. In this note, we prove that the edges of the spectral bands of a periodic Schrödinger operator are generically simple.

More precisely, on $\mathbb{R}^{n}$, consider the Schrödinger operator $H(V)=-\Delta+V$ where $V$ is a real valued, measurable, $\mathbb{Z}^{n}$-periodic potential. For the sake of simplicity, let us assume that $V$ is bounded. Then it is well known (see e.g. $[10,11]$ ) that the spectrum of $H(V)$ can be constructed in the following way. Let $\mathbb{T}^{n}$ denote the (flat) n-torus, $\mathbb{T}^{n}=\mathbb{R}^{n} / \mathbb{Z}^{n}$. In $L^{2}\left(\mathbb{T}^{n}\right)$ consider the operators

$$
H(k, V)=-(\partial+2 \pi i k)^{2}+V, k \in \mathbb{T}^{n},
$$

with common domain $H^{2}\left(\mathbb{T}^{n}\right)$. As $V$ is real-valued and bounded, for each $k, H(k, V)$ is a self-adjoint operator with eigenvalues $\left\{\lambda_{j}(k, V)\right\}_{j=1}^{\infty}$ which we list in ascending order with multiplicities, $\lambda_{j}(k, V) \leq \lambda_{j+1}(k, V)$. Then, the spectrum of $H(V)$ is given by

$$
\sigma(H(V))=\bigcup_{j=1}^{+\infty} \lambda_{j}\left(\mathbb{T}^{n}, V\right)
$$

Since the spectrum of $H(V)$ is a closed subset of $\mathbb{R}$, its complement in $\mathbb{R}$ is a countable union of disjoint open intervals, called gaps in solid state physics. The Floquet eigenvalues $\lambda_{j}(k, V)$ are continuous in $k$, and hence their ranges

$$
B_{j}=\left\{\lambda_{j}(k, V): k \in \mathbb{T}^{n}\right\}
$$

are closed intervals which are called bands of the spectrum. In this note we are concerned with the ends of the gaps, i.e. the endpoints of bands which are also endpoints of the spectrum.

Let $\lambda$ be a point in the spectrum of $H(V)$. We say that $\lambda$ is simple if there exists a single Floquet eigenvalue that assumes the value $\lambda$, i.e. there exists a unique $j \geq 1$ such that for some $k \in \mathbb{T}^{n}, \lambda_{j}(k, V)=\lambda$. Note that, since the Floquet eigenvalues are continuous with respect to $k$ and the torus $\mathbb{T}^{n}$ is compact, if $\lambda$ is simple, in a neighborhood of $\lambda$, energies either do not belong the $\sigma(H(V))$ or are simple. Moreover, if they are simple, they are assumed by the same Floquet eigenvalue.

\footnotetext{
${ }^{*}$ Received January 19, 2000.

†Département de Mathématique, Institut Galilée, U.R.A 742 C.N.R.S, Université de Paris-Nord, Avenue J.-B. Clément, F-93430 Villetaneuse, France (klopp@math.univ-paris13.fr).

$\ddagger$ Department of Mathematics, U.C.L.A., 6363 Math Sciences, 405 Hilgard Avenue, Box 951555, Los Angeles, CA 90095-1555, U.S.A. (ralston@math.ucla.edu).
} 
Let $\mathcal{V}_{k}$ denote the set of bounded $\mathbb{Z}^{n}$-periodic potentials, $V$, for which the spectrum of the Schrödinger operator $H(V)$ has at least $k$ open gaps. This is open in the topology of $L^{\infty}\left(\mathbb{T}^{n}\right)$. Our main result then states

THEOREM 0.1. The set of potentials $V$ for which the endpoints of at least $k$ gaps in the spectrum of $H(V)$ are simple is a dense, open subset of $\mathcal{V}_{k}$ in the topology of $L^{\infty}\left(\mathbb{T}^{n}\right)$.

The assumptions on the potential used in this note were chosen for the sake of technical simplicity; they are not optimal and may be relaxed (see e.g. [10]).

In dimension 1 i.e. if $n=1$, Theorem 0.1 is well known; endpoints are always simple Floquet eigenvalues (see e.g [6]). In dimension larger than 1 it was known that the lower end of the lowest band, i.e. the bottom of the spectrum, is simple (see $[1,3]$ ), and that the Floquet eigenvalue assuming this value has a non degenerate minimum at that value. As far as we know, without any further assumptions, the structure of the higher bands is unknown.

Simplicity of spectral endpoints plays an important role in many problems on periodic and perturbations of periodic Schrödinger operators. We give some references concerning the scattering theory $([2,5])$, the theory of resonances $([7])$, and the study of the counting functions for eigenvalues in a gap $([9,4])$ for perturbations of periodic Schrödinger operators.

ACKNowledgement. F.K. thanks M. Dimassi for various indications about references. F.K. thanks the Mathematics Department of the University of California at Los Angeles, where part of this work was done, for its support. J.R. thanks the Mathematics Department of the Université de Paris-Nord, where most of this work was done, for its support.

1. The proofs. We keep the notation of section 0 . Consider now $\lambda_{0}$, the upper endpoint of a gap for $\sigma\left(H\left(V_{0}\right)\right)$. Hence, there exists $m \in \mathbb{N}$ and $\varepsilon>0$ such that

$$
\lambda_{m}\left(k, V_{0}\right) \leq \lambda_{0}-\varepsilon<\lambda_{0} \leq \lambda_{m+1}\left(k, V_{0}\right)
$$

for all $k \in \mathbb{T}^{n}$. Then Theorem 0.1 is a consequence of the following.

THEOREM 1.1. There is a continuous curve, $V(t)$, in $L^{\infty}\left(\mathbb{T}^{n}\right)$ with $V(0)=V_{0}$ such that for $0<t<\epsilon$ the eigenvalue $\lambda_{m+1}(k, t)$ of $H(k, V(t))$ is simple whenever $\lambda_{m+1}(k, t)$ is sufficiently close to $\min _{k \in \mathbb{T}^{n}} \lambda_{m+1}(k, t)$.

Of course, an analogue of Theorem 1.1 holds for the lower ends of gaps. Now assume Theorem 1.1 is proven. By the continuity of the Floquet eigenvalues of $H(V)$, the simplicity of a band edge is stable under small perturbations. Starting from a potential $V_{0}$, one can then first perturb it so as to make its first gap endpoint simple, then its second gap endpoint and so on for $k$ of the open gaps for $V_{0}$, proving Theorem 0.1 .

Proof of Theorem 1.1. We begin by noting that comparison with

$$
-(\partial+2 \pi i k)^{2}+\min _{\mathbb{T}^{n}} V_{0}(x)
$$

shows that there is an $M$ such that $\lambda_{M}\left(k, V_{0}\right)>\lambda_{0}+1$ for all $k \in \mathbb{T}^{n}$. For each $k_{0} \in \mathbb{T}^{n}$ we choose a simple, closed contour in the complex plane, $C\left(k_{0}\right)$, enclosing $\left\{\lambda_{j}\left(k_{0}, V_{0}\right)\right\}_{j=m+1}^{M}$, which crosses the real axis between $\lambda_{m}\left(k_{0}, V_{0}\right)$ and $\lambda_{m+1}\left(k_{0}, V_{0}\right)$ 
and between eigenvalues of $H\left(k_{0}, V_{0}\right)$ above $\lambda_{M}\left(k_{0}, V_{0}\right)$. Then for $\left|k-k_{0}\right|$ and $\| V-$ $V_{0} \|_{\infty}$ sufficiently small, the orthogonal projection

$$
P_{k_{0}}(k, V)=\frac{1}{2 \pi i} \int_{C\left(k_{0}\right)}(H(k, V)-z I)^{-1} d z
$$

is real analytic in $k$ and $V$. Hence, we can choose an orthonormal basis $\left\{\phi_{m+1}^{\left(k_{0}\right)}, \ldots\right.$, $\left.\phi_{R\left(k_{0}\right)}^{\left(k_{0}\right)}\right\}$ for the range of $P(k, V)$ which is real analytic in $(k, V)$ on a neighborhood of $\left(k_{0}, V_{0}\right)$. Here and elsewhere we use neighborhoods in $\mathbb{T}^{n}$, and $\left|p-p_{0}\right|$ stands for the Euclidean distance between $p_{0}$ and the closest conjugate of $p$ under $\mathbb{Z}^{n}$. Thus we have a cover of $\mathbb{T}^{n}$ by open sets, and, selecting a finite sub-cover, we have open sets $\mathcal{O}_{1}, \ldots, \mathcal{O}_{P}$ with the following properties:

1. for each $\mathcal{O}_{i}$ we have an orthonormal set $\left\{\phi_{m+1}^{(i)}(k, V), \ldots, \phi_{R_{i}}^{(i)}(k, V)\right\}$ such that the $\phi$ 's are analytic in $(k, V)$ on $\overline{\mathcal{O}}_{i} \times\left\{\left\|V-V_{0}\right\|<\delta\right\}$, and

2. the span of $\left\{\phi_{m+1}^{(i)}(k, V), \ldots, \phi_{R_{i}}^{(i)}(k, V)\right\}$ contains all eigenfunctions of $H(k, V)$ belonging to eigenvalues greater than or equal $\lambda_{m+1}(k, V)$ and less than or equal to $\lambda_{0}+1$.

Next we choose $\hat{k}$ and $\phi_{0},\left\|\phi_{0}\right\|=1$, such that $H\left(\hat{k}, V_{0}\right) \phi_{0}=\lambda_{0} \phi_{0}$. Since $\left\|\phi_{0}\right\|=1$, we can choose $x_{0} \in \mathbb{T}^{n}$ such that $\left|\phi_{0}\left(x_{0}\right)\right| \geq 1$. The idea behind this proof is to set $V(t)=V_{0}-t V^{\prime}$, where $V^{\prime}$ is an approximate delta function at $x_{0}$. It requires a little work to show that $V(t)$ will have the desired properties. To simplify notation we will suppress the index $i=1, \ldots, P$ which specifies the open set $\mathcal{O}_{i}$ until the final steps of the proof. We will assume that $k \in \mathcal{O}$, one of the $\mathcal{O}_{i}$, with the understanding that all estimates which involve $k$ hold for all $\mathrm{i}$, and that all constructions are done for each $\mathrm{i}$.

First, we need the estimate

$$
\sum_{j=m+1}^{R}\left|\phi_{j}\left(x, k, V_{0}\right)-\phi_{j}\left(x_{0}, k, V_{0}\right)\right|^{2}<1 / 3
$$

for $k \in \overline{\mathcal{O}}$ when $\left|x-x_{0}\right|<\epsilon_{0}$. Since the $\phi_{j}$ 's are finite linear combinations of eigenfunctions of $H$, and multiplying these eigenfunctions by $\exp (-2 \pi i k \cdot x)$ makes them eigenfunctions of $-\Delta+V$, standard results on elliptic regularity (see, e.g. [8], Chapter 3, Section 14) imply that the $C^{\alpha}$-norms of the $\phi_{j}$ 's are uniformly bounded in $k$, giving (1.1). This would hold for $V_{0} \in L^{p}\left(\mathbb{T}^{n}\right)$ for any $p>n / 2$. We assume that $\epsilon_{0}$ is also small enough that $\left|\phi_{0}(x)\right|^{2}>2 / 3$ for $\left|x-x_{0}\right|<\epsilon_{0}$. We choose $V_{1} \in C_{0}^{\infty}\left(\left|x-x_{0}\right|<\epsilon_{0}\right)$ such that $V_{1}(x) \geq 0$ and $\int V_{1} d x=1$, and set $V(t)=V_{0}-t V_{1}$.

To see that the lowest eigenvalues of $H(k, V(t))$ above the gap have the desired behavior we will use the variational characterization (Max-Min Principle). This requires some careful choices. First we will modify $\left\{\phi_{m+1}(k, V(t)), \ldots, \phi_{R}(k, V(t))\right\}$ so that

$$
\frac{d}{d t}\left\langle\phi_{i}(k, V(t)), H(k, V(t)) \phi_{j}(k, V(t))\right\rangle=\left\langle\phi_{i}(k, V(t)), \frac{d}{d t} H(k, V(t)) \phi_{j}(k, V(t))\right\rangle
$$

for all choices of $\mathrm{i}$ and $\mathrm{j}$. Letting $\dot{f}$ denote $d f / d t$, we see that (1.2) will hold if $\left\langle\dot{\phi}_{i}, \phi_{j}\right\rangle=0$ for all $\mathrm{i}$ and $\mathrm{j}$. Setting $\tilde{\phi}_{i}=\sum_{j} u_{i j} \phi_{j}$, we have

$$
\left\langle\dot{\phi}_{r}, \tilde{\phi}_{s}\right\rangle=\sum_{j} \overline{\dot{u}_{r j}} u_{s j}+\sum_{i, j} \overline{u_{r i}} u_{s j}\left\langle\dot{\phi}_{i}, \phi_{j}\right\rangle .
$$


Hence, setting $U$ equal to the matrix with entries $u_{i j}$ and $A$ equal to the matrix with entries $-\left\langle\phi_{i}, \dot{\phi}_{j}\right\rangle,(1.2)$ will hold when the $\phi_{i}$ 's are replaced by the $\tilde{\phi}_{i}$ 's provided that

$$
\dot{U}=U A \text {. }
$$

We solve (1.3) with $U(0)=I$. Note that $U(t)$ is unitary because the orthonormality of the $\phi_{i}$ 's makes $A$ skew-symmetric, and it depends analytically on $k$ on $\overline{\mathcal{O}}$ Hence, assuming that the $\phi$ 's are the $\tilde{\phi}$ 's just constructed, both (1.1) and (1.2) hold for $V=V(t)$ when $t$ is sufficiently small. The analytic dependence of the $\phi_{i}$ 's on $V$ implies that all derivatives of $\left\langle\phi_{i}(k, V(t)), H(k, V(t)) \phi_{j}(k, V(t))\right\rangle$ with respect to $t$ are uniformly bounded in $k$ for $t$ sufficiently small. Fixing an $\mathcal{O}_{i}$ containing $\hat{k}$, we can write $\phi_{0}$ in terms of the $\phi_{j}$ 's associated with this $\mathcal{O}_{i}, \phi_{0}=\sum_{j} c_{j} \phi_{j}\left(\hat{k}, V_{0}\right)$, and then define $\phi_{0}(t)=\sum_{j} c_{j} \phi_{j}(\hat{k}, V(t))$. Then by construction

$$
\left.\frac{d}{d t}\left\langle\phi_{0}(t), H(\hat{k}, V(t)) \phi_{0}(t)\right\rangle\right|_{t=0}=-\left\langle\phi_{0}(0), V_{1} \phi_{0}(0)\right\rangle \leq-2 / 3 \text {. }
$$

Hence

$$
\left\langle\phi_{0}(t), H(\hat{k}, V(t)) \phi_{0}(t)\right\rangle \leq \lambda_{0}-2 t / 3+O\left(t^{2}\right) .
$$

On each $\mathcal{O}_{i}$, define

$$
\phi_{*}^{(i)}(k, t)=\sum_{j} \overline{\phi_{j}^{(i)}\left(x_{0}, k, V_{0}\right)} \phi_{j}^{(i)}(k, V(t)) .
$$

Given $\phi(k, t)=\sum_{j} a_{j} \phi_{j}^{(i)}(k, V(t))$, we have $\left\langle\phi(k, t), \phi_{*}^{(i)}(k, t)\right\rangle=0$ if and only if

$$
\sum_{j} a_{j} \phi_{j}^{(i)}\left(x_{0}, k, V_{0}\right)=0 .
$$

Assuming (1.5) and $\|\phi\|=1$, we have for $k \in \overline{\mathcal{O}_{i}}$

$$
\begin{aligned}
& \left.\frac{d}{d t}\langle\phi(k, t), H(k, V(t)) \phi(k, t)\rangle\right|_{t=0} \\
= & -\left\langle\phi(k, 0), V_{1} \phi(k, 0)\right\rangle \\
= & -\int_{\left|x-x_{0}\right|<\epsilon_{0}}\left|\sum_{j} a_{j}\left(\phi_{j}^{(i)}\left(x, k, V_{0}\right)-\phi_{j}^{(i)}\left(x_{0}, k, V_{0}\right)\right)\right|^{2} V_{1}(x) d x \\
\geq & -1 / 3 .
\end{aligned}
$$

Hence for $k \in \overline{\mathcal{O}_{i}}$

$$
\langle\phi(k, t), H(k, V(t)) \phi(k, t)\rangle \geq \lambda_{0}-t / 3+O\left(t^{2}\right),
$$

where the $O\left(t^{2}\right)$ is uniform in both $k$ and $\phi$ for $\|\phi\|=1$. In view of the variational characterization of the eigenvalues of $H(k, V(t))$ the estimate (1.4) implies that

$$
\lambda_{m+1}(\hat{k}, V(t))<\lambda_{0}-t / 2
$$

for $0<t<\epsilon$ with $\epsilon$ sufficiently small, and, since (1.6) bounds the minimum over the subspace defined by (4) from below,

$$
\lambda_{m+2}(k, V(t))>\lambda_{0}-t / 2
$$

for $0<t<\epsilon$, where $\epsilon$ is independent of $k$. Thus we have the desired result. This completes the proof of Theorem 1.1. 


\section{REFERENCES}

[1] S. AGmon, On positive solutions of elliptic operators with periodic coefficients in $L^{2}\left(\mathbf{R}^{n}\right)$, spectral results and extension to elliptic operators on Riemannian manifolds, in Differential equations, I. Knowles and R. Lewis, ed., North-Holland, Birmingham, 1984, pp. 7-17.

[2] F. Bentosela, Scattering from impurities in a crystal, Comm. Math. Phys., 46:2 (1976), pp. 153-166.

[3] M. Sh BIRman, Perturbations of operators with periodic coefficients, in Schrödinger Operators: Standard and Non-Standard, Dubna, 1988.

[4] M. SH. BIRMAN, The discrete spectrum in gaps of the perturbed periodic Schrödinger operator II. Nonregular perturbations, Algebra i Analiz, 9:6(1997), pp. 62-89.

[5] M. SH. BIRMAN AND D. R. YAFAEV, The scattering matrix for a perturbation of a periodic Schrödinger operator by decreasing potential, Algebra i Analiz, 6:3 (1994), pp. 17-39.

[6] M. Eastham, The Spectral Theory of Periodic Differential Operators, Scottish Academic Press, Edinburgh, 1973.

[7] C. GÉRARD, Resonance theory for periodic Schrödinger operators, Bulletin de la Société Mathématique de France, 118 (1990), pp. 27-54.

[8] Olga A. Ladyzhenskaya and Nina N. Ural'tseva, Linear and Quasilinear Elliptic Equations, Academic Press, New York, 1968. Translated from the Russian by Scripta Technica, Inc. Translation editor: Leon Ehrenpreis.

[9] G. D. RAĬKOV, Eigenvalue asymptotics for the Schrödinger operator with perturbed periodic potential, Invent. Math., 110:1 (1992), pp. 75-93.

[10] M. Reed And B. Simon, Methods of Modern Mathematical Physics, Vol IV: Analysis of Operators. Academic Press, New-York, 1978.

[11] J. SJöstrand, Microlocal analysis for periodic magnetic Schrödinger equation and related questions, in Microlocal analysis and applications, Lecture Notes in Mathematics 1495, Springer Verlag, Berlin, 1991. 
